\title{
AN INNOVATIVE GEOCENTRIC DECISION SUPPORT SOLUTION TO COMPREHENSIVE PLANNING, DESIGN, OPERATION, AND MANAGEMENT OF URBAN DRAINAGE SYSTEMS
}

\author{
Paul F. Boulos , Misgana K. Muleta , Chun-Hou Orr and Jun Je Ro
}

\begin{abstract}
Geographic Information System (GIS) is quickly becoming a critical component to develop and sustain asset management for today's wastewater utilities as most of their data is geographically referenced. This technology offers sophisticated data management and spatial analysis capabilities that can greatly improve and facilitate urban drainage infrastructure modeling and analysis applications. This paper presents a comprehensive GIS-based decision support system that integrates several technologies for use in the effective management of urban stormwater collection systems. It explicitly integrates ESRI ArcGIS geospatial model with advanced hydrologic, hydraulic, and water quality simulation algorithms, nature-based global optimization techniques including genetic algorithms for design and calibration of stormwater management models, automated dry weather flow generation and allocation, and automated subcatchment delineation and parameter extraction tools to address every facet of urban drainage infrastructure management. The geocentric interface allows seamless communication among the various modules. The resulting decision support system effortlessly reads GIS datasets, extracts necessary modeling information, and automatically constructs, loads, designs, calibrates, analyzes and optimizes a representative urban drainage model considering hydrologic and hydraulic performance requirements. It also makes it easy to run, simulate and compare various modeling scenarios, identify system deficiencies, and determine cost-effective physical and operational improvements to achieve optimum performance and regulatory compliance. These combined capabilities provide favorable geospatial environment to assist wastewater utilities in planning, designing, and operating reliable systems and in optimizing their capital improvement programs.
\end{abstract}

\section{INTRODUCTION}

Comprehensive modeling of sewer collection systems is essential to develop reliable and cost-effective remedial solutions for enhancing system integrity and performance, restoring and maintaining needed capacity, avoiding backups and overflows and meeting environmental regulations to improve public health and safety. However, modeling of urban stormwater and sewer collection systems requires 
extensive spatial and temporal data due to the complexity of the governing processes and the heterogeneity of watershed properties and flow paths and conduits found in developed and undeveloped urban areas. These features are geographic in nature and suggest the need for an efficient spatial data management and analysis tool such as a Geographic Information System (GIS). GIS provides functions for development and preparation of accurate spatial information for input (pre-processing) to urban drainage system models. It also facilitates post-processing spatial analysis and graphical output display for evaluating results. Therefore, the marriage of mathematical stormwater models and GIS would ensure that sound engineering solutions are drawn efficiently and effectively in the design, planning, maintenance, and operation of wastewater collection systems (Miles and Ho, 1999). These benefits are now being realized by wastewater utilities and municipalities in managing their sanitary, storm and combined sewer system infrastructures.

Shamsi (2002) distinguishes three different methods a GIS can be linked to simulation (mathematical) models: interchange, interface, and integration, listed in order of complexity and versatility. The interchange method employs a batch process to transfer data between the GIS and simulation model. Both GIS and the simulation model are run separately and no direct link exists between the two systems. The interface method provides a direct link to exchange information between the GIS and the simulation model with customized pre- and post-processing functions added to the GIS. However, the interface method also requires the simulation model to run independently from the GIS. The integration method represents the closest relationship between the GIS and the simulation model. The method combines both the GIS and the modeling functions in one complete seamlessly integrated package. It provides the basis for a comprehensive decision support system for the effective management of urban drainage systems.

In addition to ensuring seamless integration of GIS with a mathematical model that simulates the hydrology, hydraulics and water quality of urban drainage systems, a comprehensive decision support model should have: tools that facilitate watershed delineation and extraction of input parameters from digital topographic, soil and land use data; a calibration model that identifies optimal parameter values so that model predictions closely match observed data; a design model that determines cost-effective solutions to eliminate flooding and associated pollution problems; a load allocation tool that computes wastewater loads entering the collection system at various locations; and the capability to analyze important water quality parameters such as hydrogen sulfide whose improper management could cause serious problems including loss of life (Nicklow et al, 2004).

This paper presents an integration of the USEPA storm water management model (SWMM5) for hydrologic, hydraulic, and water quality simulation with GIS (ArcGIS, ESRI, Redlands, California) and an efficient variation of genetic algorithms (GA) optimization technology to address every facet of urban wastewater management activities. The resulting software system, called InfoSWMM Suite, effortlessly reads GIS data, extracts necessary modeling information such as subcatchment parameters, and automatically constructs, loads, calibrates, designs, analyzes and optimizes a representative model considering hydraulic, water quality and hydrologic management and operational requirements. It also makes it easy to simulate various scenarios, 
identify deficiencies, and determine cost-effective improvements for optimum performance. It is a single software platform that addresses the requirements of both wastewater utility engineers and GIS professionals and provides an informative structured framework for complete sewer model construction, analysis, optimization, and results presentation. These combined capabilities provide a consistent geospatial environment to assist wastewater utilities in planning, designing, and operating reliable systems, evaluating the effectiveness of best management practices (BMPs), and in optimizing their capital improvement programs.

\section{A GEOSPATIAL DECISION SUPPORT SYSTEM - INFOSWMM SUITE}

The InfoSWMM Suite decision support system (Boulos, 2005) approaches wastewater collection system modeling from a GIS-centric point of view and works to minimize the efforts needed to create, analyze and optimize a drainage system model. Built atop of ArcGIS, InfoSWMM Suite is a full-featured sewer collection system modeling and optimization software solution. As shown in Figure 1, the core of the geospatial decision support system is the central geodatabase for storage, manipulation, and display of the collective simulation model results. No data conversion is required and the user interacts directly with the GIS (there is no need to exit the GIS to edit data or run an analysis). The geospatial user interface initializes the GIS environment, creates all the necessary input files, builds network topology, sets hydrologic, hydraulic and water quality parameters and optimization constraints, runs and compares any number of modeling scenarios, and displays and tabulates results.

The InfoSWMM Suite hosts, in a unifying framework, the variety of processes required for loading, calibrating, analyzing, and optimizing wastewater collection network models. These are divided into five embedded simulation modules for automated dry weather flow (DWF) estimation and allocation (DWF Allocator); subcatchment parameter extraction (Subcatchment Manager); hydrologic, hydraulic and water quality analysis (Analyzer); optimal hydraulic calibration (Calibrator); and least-cost design (Designer). It combines the ability to accurately build network topology, prepare requisite data, conceive and evaluate multiple scenarios, execute optimization runs, and provide both hardcopy reporting and graphical output display for evaluating and presenting results.

The optimization modules are predicated on the use of the fast messy genetic algorithm (fmGA), which is one of the most competent types of GA delivering reliable solutions in sub-quadratic time (Boulos et al, 2003). They operate jointly with the hydrologic, hydraulic and water quality solver (simulation module) to provide direct feedback on impacts of computed solutions on wastewater collection system performance. Both modules are applied in a convergent scheme as follows. The optimization model initially creates a population of candidate solutions, which are then passed to the network solver to analyze the hydrologic, hydraulic and water quality performance in satisfying the imposed constraints. The computed solutions are then passed to the decision support system for use in quantifying the objective function and any constraint violations. This information is then used to produce a new population with better-fit members which is then passed back to the network solver for subsequent evaluation. The iteration continues until a specified level of convergence is reached. Because the 
decision support software was built using an open-system architecture approach, it can be easily expanded to incorporate additional simulation and optimization modules planned in the future.

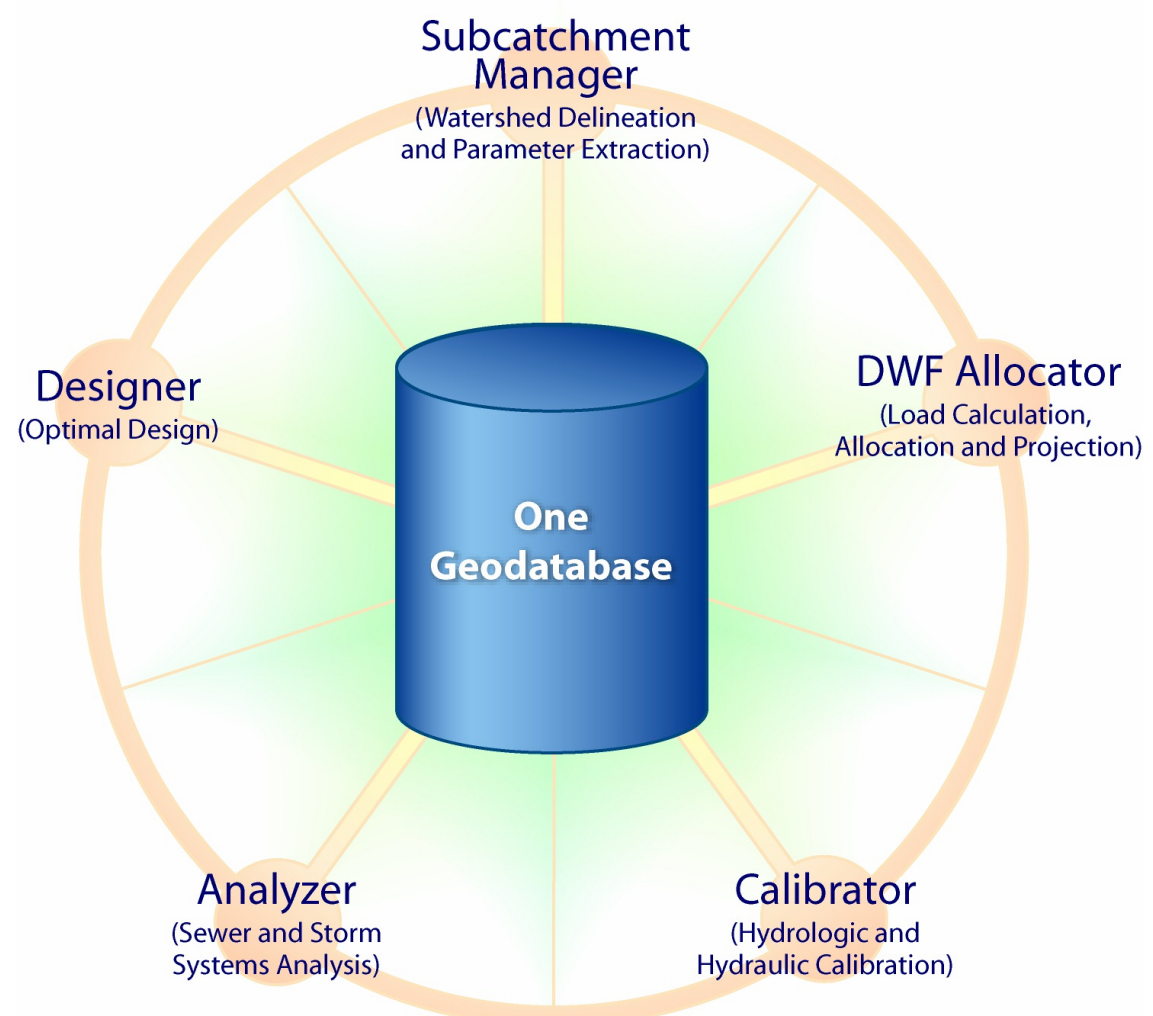

Figure 1: The InfoSWMM suite model architecture.

Each module of the geospatial decision support system is described below.

\subsection{Drainage System Simulation Model (Analyzer)}

InfoSWMM Analyzer uses an extended version of the EPA SWMM5 (Rossman, 2005) model to solve urban drainage hydrology, hydraulics and water quality. It can model the entire land phase of the hydrologic cycle as applied to urban stormwater and wastewater collection systems. The model can perform single event or long-term (continuous) rainfall-runoff simulations accounting for climate, soil, land use, and topographic conditions of the watershed. In addition to runoff quantity, it can simulate runoff quality including buildup and washoff of pollutants from primarily urban watersheds. Once runoff quantity and quality is simulated and wastewater loads at receiving junctions are determined, the routing portion of InfoSWMM Analyzer can transport the flow using steady, kinematic wave or dynamic wave routing, through a conveyance system of pipes, channels, storage/treatment devices, pumps, and hydraulic regulators such as weirs, orifices, and other outlet types. It can accurately simulate complex flow conditions including backwater effect, flow reversal, and pressurized flow. Because it couples together the solution for both water levels at nodes and flow in conduits, Analyzer can be applied to any general network layout, even those containing 
multiple downstream diversions and loops. The model offers advanced Real-Time Control (RTC) scheme for the operational management of hydraulic structures. It can also model hydrogen sulfide buildup and corrosion potential as well as street overland flows and stormwater drainage inlets.

\subsection{Subcatchment Parameters Extractor (Subcatchment Manager)}

Proper delineation of watersheds is critical for accurate stormwater modeling applications. The Subcatchment Manager (Figure 2) provides a suite of geospatial tools for defining watershed data in a GIS and then using the information to directly create and manage hydrologic and hydraulic models, or as a support utility for data development from topographic, soil, and land use maps. To simulate runoff quantity InfoSWMM requires subcatchment inputs including area, imperviousness, slope, width (a shape factor), and infiltration parameters for Horton, the Green-Ampt, or the Curve Number methods for individual subcatchments in the system. The Subcatchment Manager is designed to utilize digital topographic data such as DEM, TIN, contours, and point elevation to delineate watersheds, streams/channels, and subwatersheds, and to extract the hydrologic parameters listed above for each subcatchment from topographic, land use, and soil maps available for the watershed.

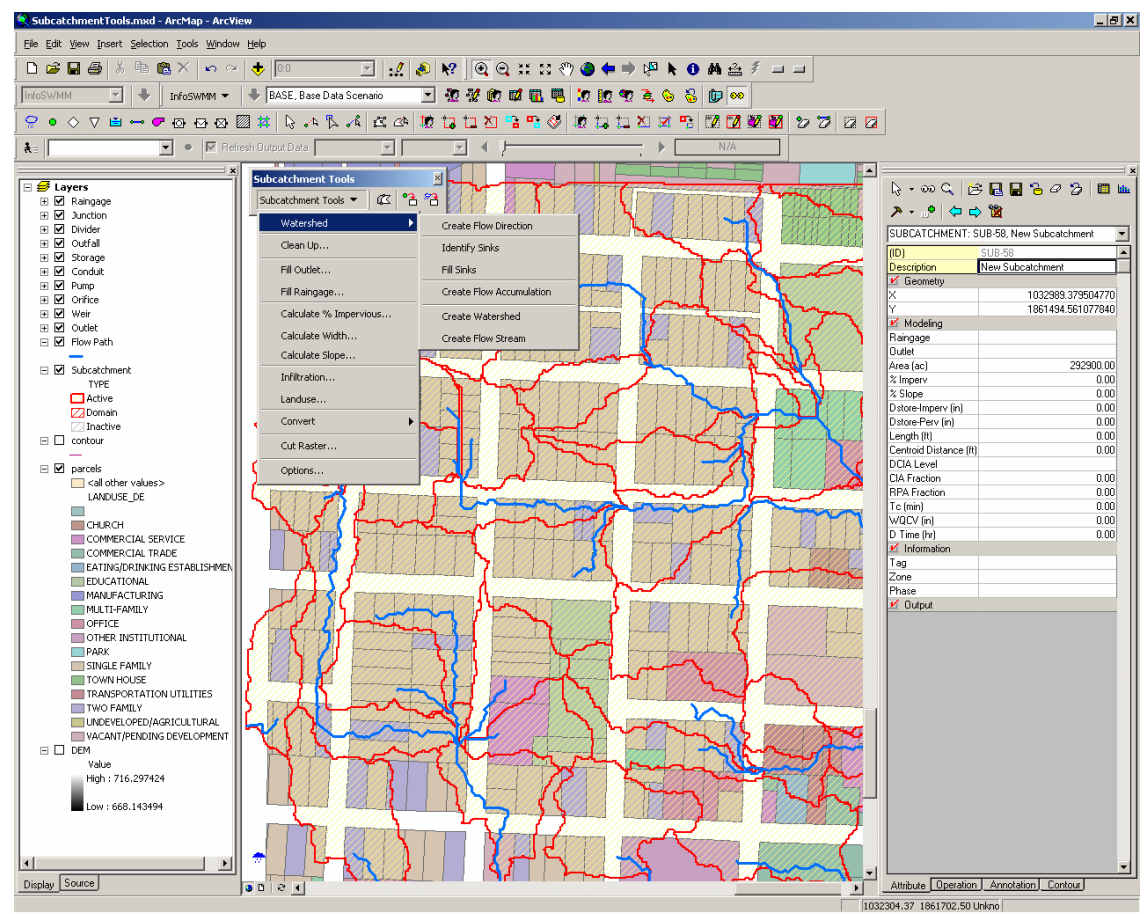

Figure 2: The InfoSWMM subcatchment manager module.

Using the Subcatchment Manager one can delineate a watershed for an urban drainage system; subdivide the watershed into subwatersheds/subcatchments; create streams (channels) and converts these channels into conduits of any shape; create an outlet for the drainage system; create rainfall time series from radar data; assign raingage to subcatchments based on their geographic proximity; determine subcatchment parameters such as width (using several methods), area, and slope from DEM, TIN, 
contours or point elevations; extract percent imperviousness and subcatchment land use types from digital land use data; determine infiltration parameters from digital soil map, and develop depth versus area curve for detention ponds from DEMs. In other words, with the help of Subcatchment Manager alone one can build a working urban drainage system model from topographic, land use, soil, and radar data. The model enables water resources engineers to devote more time to understanding problems and evaluating solutions instead of performing drudgery.

\subsection{DWF Load Estimation and Allocation (DWF Allocator)}

Determining wastewater loads and their spatial distribution throughout the drainage system model is a key element of wastewater collection system modeling. DWF data can be derived from many sources including flow metering, water consumption records, system flows, and estimates from consumer characteristics, such as population, land use, traffic counts, or other parameters. These data are assigned as DWF values at individual junctions within the collection system model where selected junctions receive flows from distinct service areas. Generally, the average DWF loads are first estimated for all junction nodes, and temporal (e.g., diurnal and seasonal variation) of the loads are then adjusted for various land use categories such as residential and commercial areas. The DWF Allocator module was designed to assist in the process of generating and allocating network DWF loads for existing system conditions and for various planning horizons. It offers seven methods for processing geometric polygons to compute and load network models based on load type, location, and variation (Figure 3). These are:

1. Geocoded meter billing data (meter DWF database)

2. Polygon intersection - spatial intersection of multiple polygon layers

3. Polygon extraction - spatial summation of DWF category area polygons

4. Closest Junction - allocate loads to the nearest junction

5. Closest Conduit - allocate loads to the nearest conduits

6. Meter-Junction Allocation - user-defined assignment of meters to junctions

7. Meter-Conduit Allocation - user-defined assignment of meters to conduits

The first method uses geographic information system (GIS) layers to allocate geocoded load data. Here, the DWF Allocator determines the flow at each network junction by identifying and summing all the customers/meters in its associated load area polygon. These loads include customers such as residential, commercial, industrial, schools, parks, golf courses, hospitals, etc. which are drawn directly from their spatially located water consumption records. In the second method, the DWF Allocator calculates flows based on direct spatial intersection between load categorization polygons (e.g., land use polygons, population polygons, pressure zone polygons, TAZ polygons, census tract polygons, meter route polygons, and others) and load area coverage polygons (service area polygons). In the third method, junction flows are calculated by summing individually assigned load category polygons. The fourth and the fifth methods work in conjunction with geocoded customer/meter data. The fourth method locates the junction closest to the meter by using advanced search algorithms and then allocates loads. In the fifth method, search algorithms are used to locate the closest conduit to each meter. Loads are then assigned to the nearest junction on either side of the conduit or divided 
based on a distance-weighted approach. The last two methods are similar to the closest conduit and the closest junction methods, except the user is able to determine which meters will be assigned to which junctions and conduits in the system. The meterjunction and the meter-conduit allocation methods allow the user the freedom to graphically determine which meters are tributary to which junctions and conduits in the sewer collection system.

These comprehensive capabilities give practicing engineers considerable flexibility to effectively utilize their knowledge and experience and leverage existing GIS data investments to strategically define/forecast their network DWF load estimation for various planning horizons in their master planning effort.

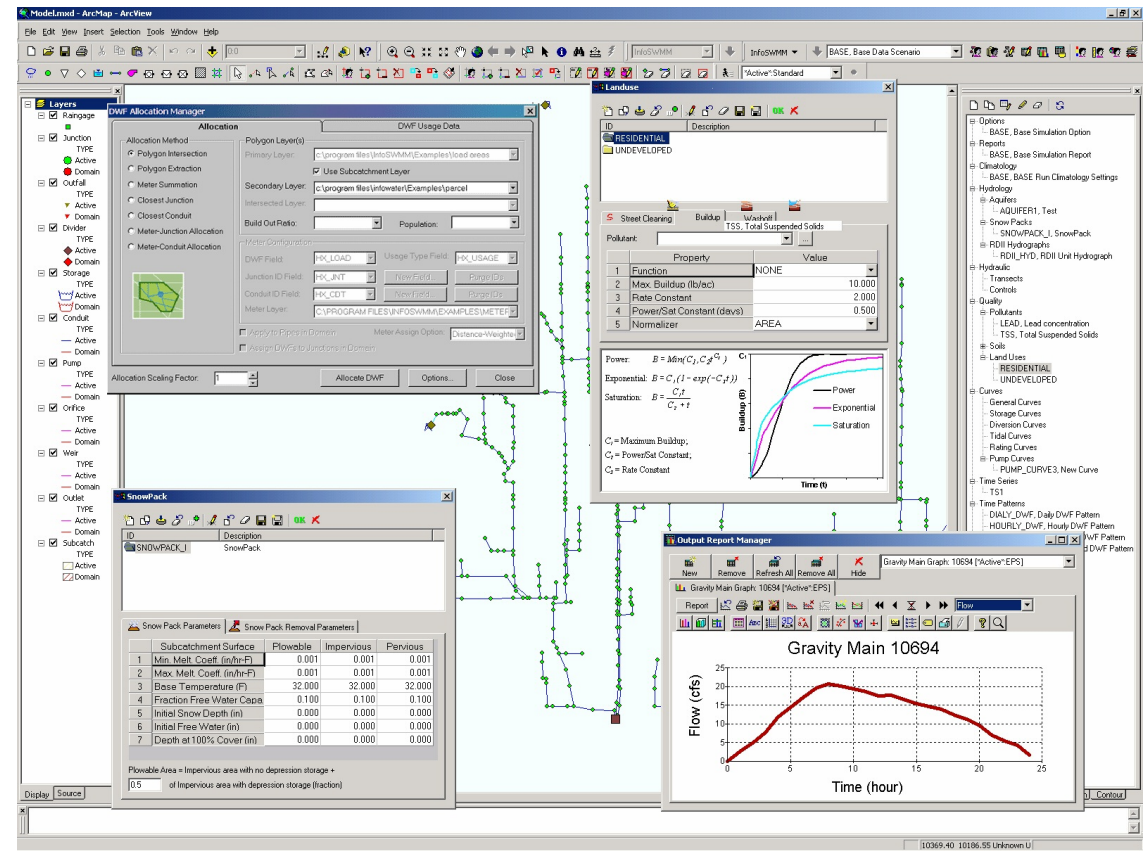

Figure 3: The InfoSWMM DWF load allocation module.

\subsection{Network Model Calibration (Calibrator)}

After a network model is properly constructed, it must be calibrated to the physical system so that model predictions can be interpreted with confidence (Muleta and Nicklow, 2005). Traditionally, calibration of an urban drainage network model was an exhaustive trial-and-error process of adjusting the model input parameters until model results coincide with field observations. However, since there is a vast number of combinations of parameter values that need to be considered, manual evaluation of all options through trial-and-error is unlikely to be practically feasible or manageable, and even knowledgeable modelers often fail to obtain good results. As a result, network model calibration has generally been neglected or done haphazardly. InfoSWMM Calibrator (Figure 4) fully automates and simplifies this manual process, making urban drainage model calibration significantly easier and the model more reliable.

In order to improve the reliability of network models as well as eliminate the need for trial-and-error calibration methods, the calibration problem is cast as optimization 
problem and solved using GAs and PSO. Any combination of flow, depth, and/or velocity measurements could be used as observation, and the decision parameters are classified into five distinct groups (subcatchment group, soil group, aquifer group, RDII group, and conduit group). The Subcatchment group represents subcatchment parameters and groundwater parameters including area, width, percent imperviousness, slope, Manning's $\mathrm{N}$ for pervious and impervious subareas, depression storage for pervious and impervious subareas, Colorado Urban Hydrograph Procedure parameters such as length, centroid distance, CIA fraction, RPA fraction, lag time for Natural Resources Conservation Service's dimensionless and triangular unit hydrograph method, groundwater flow coefficient, surface water flow coefficient, groundwater flow exponent, surface water flow exponent, surface-groundwater interaction coefficient). The soil group contains infiltration parameters including Horton's parameters such as maximum infiltration rate, minimum infiltration rate, decay rate, drying time, maximum volume; Green-Ampt parameters such as suction, conductivity, initial deficit and Curve Number parameters such as curve number, conductivity, and drying time. The aquifer group comprises aquifer parameters including porosity, wilting point, field capacity, conductivity, conductivity slope, tension slope, upper evaporation fraction, lower evaporation depth, bottom elevation, water table elevation, and unsaturated zone moisture. The RDII (Rainfall Driven Infiltration and Inflow) group represents RDII unit hydrograph parameters including $\mathrm{R}, \mathrm{T}$, and $\mathrm{K}$ for short-term, medium-term, and longterm responses, and the conduit group represents conduit parameters including the Manning's roughness coefficient.

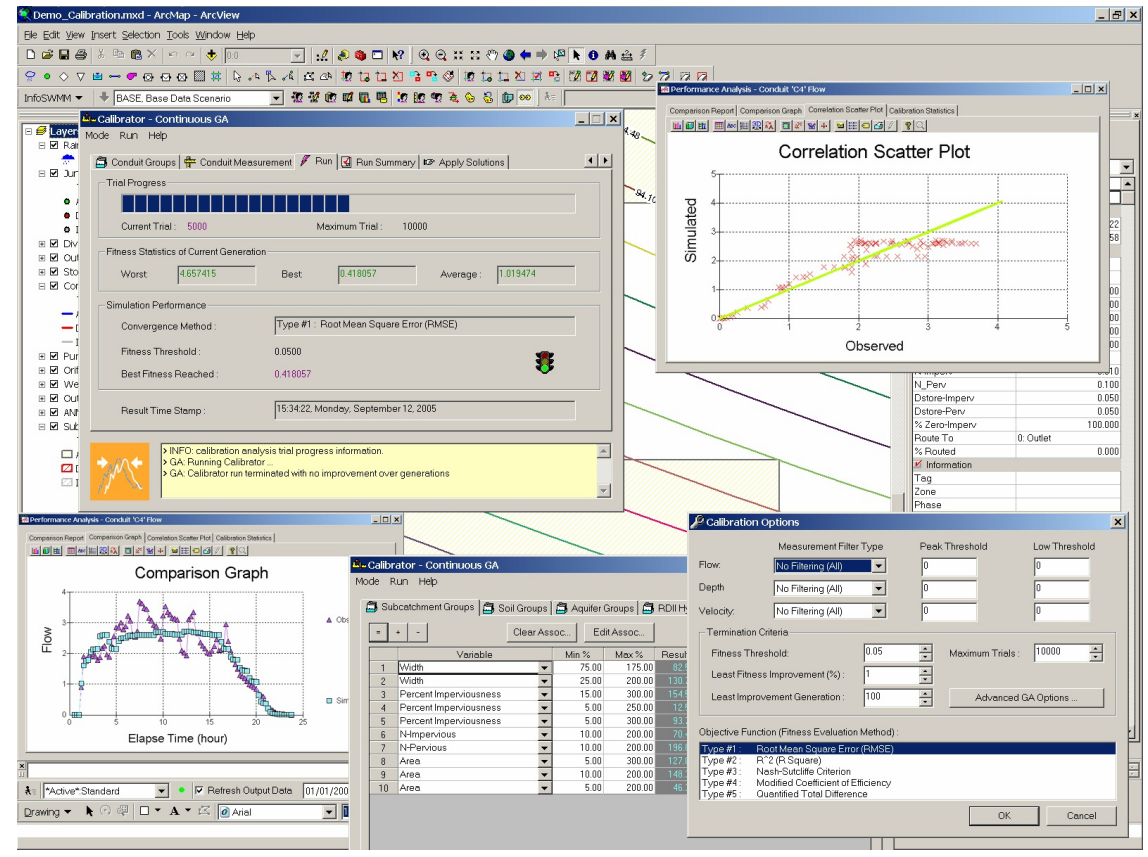

Figure 4: The InfoSWMM optimal calibration module.

InfoSWMM Calibrator provides multiple goodness-of-fit evaluation criteria including Root Mean Square Error, Nash-Sutcliffe Efficiency Criterion, Modified Coefficient of Efficiency, R-Square, and deviation in total volume of observed and simulated values. Any one of these evaluation criteria could be used to identify set of model parameters so that model simulations best fit the entire time series of the measured data, or peak 
values such as peak flows (measured values higher than specified threshold), or low values such as low flows (values lower than specified threshold). Stopping criteria could be any one or more of fitness threshold (e.g., R-Square greater than 0.99), tolerance of the differences between observed and simulated values, the maximum number of model simulations, and improvement in the fitness criteria within specified number of generations. Calibration results are presented graphically (scatter plot and time series plot), tabularly, and in the form of statistical summary.

\subsection{Network Design (Designer)}

Cost-effective design of urban drainage systems is one of the most pressing problems in engineering practice. Mitigation of the adverse impacts of flooding and the associated pollution problems is normally achieved using engineering measures such as placement of stormwater detention ponds, improving conveyance capacity by pipe upsizing, boosting pumping capacity, and by adding various Combined Sewer Overflow (CSO) structures to the collection system. The objective is to minimize system improvement costs while satisfying regional environmental regulations for runoff quantity and quality control. InfoSWMM Designer (Figure 5) solves the design problem using genetic algorithm optimization.

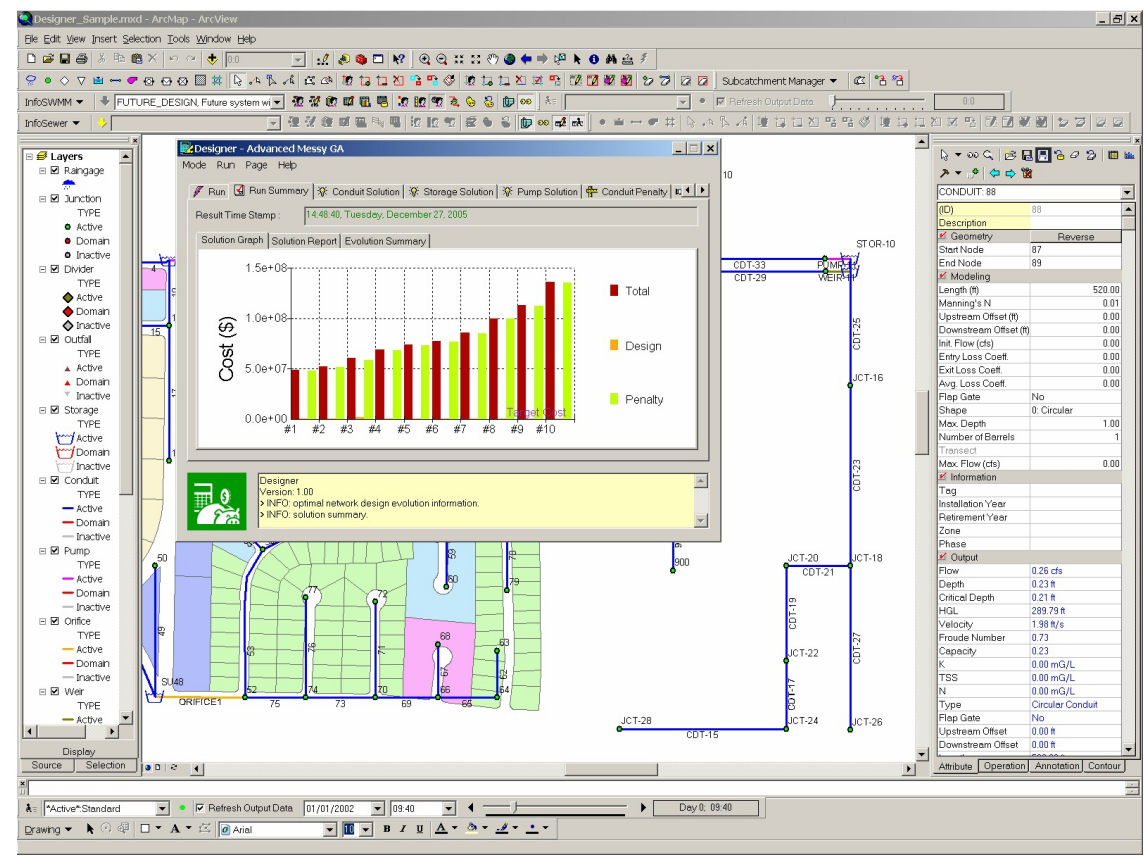

\section{Figure 5: The InfoSWMM optimal design module.}

The design problem is cast as an optimization problem and solved using the fmGA. The optimal solution is selected from a combination of pipe slope and size, storage, pumping, and new piping to eliminate unwanted sewer overflows and achieve targeted system performance requirements. Performance criteria include maximum allowable depth to diameter ratio, minimum and maximum pipe velocities and slopes, and maximum head loss for force mains. Cost data is specified for each option depending, for example, on the size and location the conduit, size and location of detention pond, 
and size and type of pump. Cost data could vary with geographical location of the network element. Results include top ten design solutions and associated costs presented graphically and in tabular form along with statistical summary.

\section{CONCLUSIONS}

Today's wastewater utilities are discovering a wide variety of application areas of GIS technology. In particular, GIS information is critical to urban drainage system planning and analysis. InfoSWMM has been presented as a decision support system for comprehensive sewer collection system management. Built entirely atop of ArcGIS, the software seamlessly integrates GIS features and functionalities with sewer collection system hydraulic, hydrologic, and water quality simulation and genetic algorithm-based optimization technology. The integrated system allows accurate urban drainage network model construction and simulation, and provides a reliable and effective means for decision makers to quickly assess and address the implications of alternative design and operational changes on system performance. As the current trend towards the creation of comprehensive geodatabases continues, the proposed decision support system would be even more useful for planning and managing sewer collection systems. It allows a wide range of cost-effective network alternatives to be modeled, analyzed, contrasted, and evaluated, providing sound guidance to wastewater utility managers to effectively optimize their capital improvement programs, keep their systems operating well in the future, and forge closer ties with their customers. It is managing complexity through simplicity.

\section{REFERENCES}

Boulos, P.F., 'InfoSWMM Suite - Users Guide', MWH Soft, Inc., Pasadena, CA, 2005. Boulos, P.F., et al., 'Using Genetic Algorithms for Water Distribution System Optimization', In Proceedings of the ASCE Environmental and Water Resources Institute's (EWRI's) World Water \& Environmental Resource Congress, May 20-24, Orlando, FL, 2001.

Miles, S.B., and Ho, C.L., 'Applications and Issues of GIS as a Tool for Civil Engineering Modeling', Journal of Computing in Civil Engineering ASCE, 13(3), 1999, 144-152.

Muleta, M.K., and Nicklow, J.W., "Sensitivity and uncertainty analysis coupled with automatic calibration for a distributed watershed model." J. of Hydrology, Elsevier, 306: 2005, 127-145.

Nicklow, J.W., Boulos, P.F., and Muleta, M.K., 'Comprehensive Sewer Collection Systems Analysis Handbook for Engineers and Planners', 1st ed., MWH Soft, Inc. Publ., Pasadena, CA, 2004, 273 pp.

Rossman, L.A., "Stormwater Management Model User's Manual, Version 5", U.S. EPA, Cincinnati, OH, 2005.

Shamsi, U.M., 'GIS Tools for Water, Wastewater, and Stormwater Systems', ASCE Press, Reston, VA 375A, 2002, 273 pp. 